\title{
V3 - IMMUNIZATION WITH RECOMBINANT, PLANT-PRODUCED YELLOW FEVER VIRUS ENVELOPE (E) PROTEIN VACCINE CANDIDATES IN RHESUS MACAQUES
}

$\underline{\text { Rosane Cuber Guimarães }}^{1}$, Andrea Nazare Monteiro Rangel da Silva ${ }^{1}$, Luciane Pinto Gaspar $^{1}$, Marisol Simões ${ }^{1}$, Patrícia Cristina da Costa Neves ${ }^{1}$, Gisela Trindade ${ }^{1}$, Renato Marchevsky $^{1}$

1. Bio-Manguinhos, Oswaldo Cruz Foundation, Technological Development, Rio de Janeiro Brazil

Objectives: This work describes a non-human primate immunogenicity study and the humoral and cellular response for the YFE antigen as part of the development of a subunit vaccine against the Yellow Fever envelope (E) protein utilizing the plant-based expression platform.

Methods: This study included 20 animals and two formulations (one each of YF-E alone and fusion to lichenase) were selected based on immunogenicity studies in mice. Monkeys were immunized intramuscularly with $30 \mu \mathrm{g}$ of YFE-1T and YFE-2 plus Alhydrogel ${ }^{\mathrm{TM}}$ three times at 30-days interval. One group was immunized intramuscularly with a single dose of 17DD vaccine. The Neutralizing Antibody Titers (Log $10 \mathrm{mIU} / \mathrm{mL}$ ) were assayed by PRNT 30, 60, 90 days after first immunization and after challenge with 17DD vaccines in all groups (104 DPI). Viremia and RNAemia after challenge of all groups were assayed by titration and qPCR. We also measured yield of total IgG against yellow fever E protein; Avidity Index (AI) of $\mathrm{IgG}$ antibodies against $\mathrm{E}$ protein in rhesus sera; and magnitude of cellular immune response against E protein measured by IFN- $\gamma$ ELISpot. The Functional profile of CD4+ $\mathrm{T}$ cell and CD8+ $\mathrm{T}$ cell responses after challenge with 17DD in rhesus monkeys vaccinated with YF 17DD, YFE-1T and YFE2E was assessed. We carried out multi-parameter ICS at day 104 (14 days after challenge with YF 17DD virus) to determine the ability of protein E-specific CD4 ${ }^{+}$T-cells and $\mathrm{CD}^{+}$T-cells to degranulate (CD107a) and secrete IFN- $\gamma$, TNF- $\alpha$,and/or IL-2. The antigen stimuli in this assay consisted of recombinant E protein at $20 \mu \mathrm{g} / \mathrm{mL}$.

Results and Conclusion: All monkeys seroconvert after the second dose of YFE-1T and YFE2E. After challenge, viremia could be detected in 3 animals inoculated with 
recombinant proteins that indicates a non-sterile immunity even using an attenuated virus for the challenge. Viremia and RNAemia after challenge is lower in mock group when compared with the clean group, indicating an influence of the adjuvant alone. For both recombinant proteins there is a booster effect after the second dose, however there is a decline in day $90 \mathrm{IgG}$ levels when compare to day 60 levels. Increase in samples' avidity index (AI) in the groups immunized with both recombinant proteins and 17 DD group was observed. In this study, it was possible to demonstrate that both recombinant proteins were able to stimulate $\mathrm{T}$ cell response specific for the protein E. Although the groups immunized with recombinant proteins receive two more doses, it was not possible to observe an increase in the number of clones responders on days 60 and 90.The ability of protein E-specific CD4 ${ }^{+} \mathrm{T}$-cells and $\mathrm{CD}{ }^{+} \mathrm{T}$-cells to degranulate (CD107a) and CD4 TNF- $\alpha$ production are the most impaired functions when compared 17DD with recombinant proteins 